\title{
Schimke Immunoosseous Dysplasia
}

National Cancer Institute

\section{Source}

National Cancer Institute. Schimke Immunoosseous Dysplasia. NCI Thesaurus. Code C135087.

An autosomal recessive condition caused by mutation(s) in the SMARCAL1 gene, encoding SWI/SNF-related matrix-associated actin-dependent regulator of chromatin subfamily A-like protein 1. It is characterized by short stature, intrauterine growth restriction, microdontia, depressed nasal bridge, skeletal dysplasia, immune complex nephritis and immune deficiency. 